\title{
Clinical Features and Outcome in Adult Cases of Tuberculous Meningitis in Tertiary Care Hospital in Antananarivo, Madagascar
}

\author{
Mihaja Raberahona, ${ }^{1}$ Rivonirina Andry Rakotoarivelo, ${ }^{2,3}$ Tiana Razafinambinintsoa, ${ }^{1}$ \\ Radonirina Lazasoa Andrianasolo, ${ }^{1,4}$ and Mamy Jean de Dieu Randria ${ }^{1}$ \\ ${ }^{1}$ Service des Maladies Infectieuses, Hôpital Universitaire Joseph Raseta Befelatanana, Antananarivo, Madagascar \\ ${ }^{2}$ Service des Maladies Infectieuses, CHU Tambohobe, Fianarantsoa, Madagascar \\ ${ }^{3}$ Université de Fianarantsoa, Fianarantsoa, Madagascar \\ ${ }^{4}$ Programme National de Lutte contre la Tuberculose (PNLT), Ministère de la Santé Publique, Antananarivo, Madagascar \\ Correspondence should be addressed to Mihaja Raberahona; raberahona@gmail.com
}

Received 10 December 2016; Accepted 5 March 2017; Published 15 March 2017

Academic Editor: Valeria Rolla

Copyright ( 2017 Mihaja Raberahona et al. This is an open access article distributed under the Creative Commons Attribution License, which permits unrestricted use, distribution, and reproduction in any medium, provided the original work is properly cited.

\begin{abstract}
Purpose. We aimed to describe and to assess prognosis factors in tuberculous meningitis in adult patients. Methods. We performed a retrospective study of case records of adult patients. Patients classified as definite, probable, or possible tuberculous meningitis according to standardized definition criteria were included and assessed in the study. Results. Seventy-five patients were included in the study. Tuberculous meningitis was classified as definite in 8 (10.7\%), probable in 44 (58.7\%), and possible in 23 patients (30.6\%). HIV was found in $3 \%$ of patients. Patients were in advanced stages at admission in $82.7 \%$. Median duration of symptoms prior to admission was 3 weeks (IQR: 2-5). Median time to diagnosis following admission was 5 days (IQR: 3-8). Median CSF WCC was 75 per $\mathrm{mm}^{3}$ with lymphocytic predominance in 38 cases (52.8\%). Median CSF glucose level was $1.48 \mathrm{mmol} / \mathrm{L}$ and median CSF protein level was $1 \mathrm{~g} / \mathrm{L}$. Mortality rate was $28 \%$. Age $\geq 35$ years (aOR: 4.06; 95\% CI: 1.16-14.26) and coma (aOR: 12.98; 95\% CI: 1.13-149.16) predicted inpatient mortality. Conclusion. Most of the patients experienced more than 3 weeks of diagnostic delay prior to admission. Mortality was high and occurred early after admission. Age and coma were identified as independent prognosis factors.
\end{abstract}

\section{Introduction}

Madagascar ranks among countries where tuberculosis is highly endemic. In 2013, the World Health Organization (WHO) estimated the prevalence of tuberculosis to be 442 per 100,000 population (95\% CI: $222-735$ ) and incidence to be 234 per 100,000 population (95\% CI: 139-280). Estimated mortality rate was 46 per 100,000 population (95\% CI: 19-84). A total of 19,010 new cases of pulmonary tuberculosis and 4,964 new cases of extrapulmonary tuberculosis were notified [1]. Tuberculous meningitis (TBM) accounts for approximately $1 \%$ of all forms of tuberculosis and $5.1 \%$ of extrapulmonary tuberculosis $[2,3]$. However, TBM is associated with both high rate of mortality and morbidity. Indeed, mortality rate ranges between $40.3 \%$ and $87.9 \%$ in Africa and neurological sequelae may affect up to $78.5 \%$ of surviving patients during follow-up $[4,5]$. Moreover, the diagnosis of TBM is difficult due to nonspecific clinical presentation and lack of sensitivity of microbiological confirmation tests [6]. In addition, in the context of resource-limited countries as Madagascar with weak health system and limited access to laboratory tests, diagnosis of TBM remains a challenge. Thereby, TBM is probably under diagnosed and not well known in Madagascar.

In this study, we aimed (i) to describe clinical, laboratory, and radiological findings and (ii) to assess prognosis factor in adult patients with TBM. 


\section{Methods}

2.1. Study Setting and Design. We conducted this singlecenter study in the Infectious Diseases Unit of the University Hospital Joseph Raseta Befelatanana, Antananarivo, Madagascar. Antananarivo is the capital and the largest city of Madagascar. The University Hospital Joseph Raseta Befelatanana is a tertiary care reference hospital dedicated to medical specialties with approximately 350 beds. About 60 and 80 patients are admitted monthly in the Infectious Diseases Unit. We retrospectively reviewed the case records of adult patients (age $\geq 16$ years) with TBM admitted between 2007 and 2014.

2.2. Case Definition. Patients classified as definite, probable, or possible TBM according to standardized case definition proposed by Marais et al. [7] were included in this study. Thereby, patients were considered as having a definite TBM if evidence of Mycobacterium tuberculosis (MTB) is found in the cerebrospinal fluid (CSF) either by detection of acid-fast bacilli (AFB) on microscopy or by culture or by molecular technique such as nucleic acid amplification (NAA) test. The definition of probable or possible TBM depends on diagnostic score based on clinical, CSF, and radiological criteria. A maximum of 6 points is allocated to clinical criteria which includes the following items: symptom duration of more than 5 days (4 points), systemic symptoms suggestive of tuberculosis (2 points), history of recent close contact with an individual with pulmonary tuberculosis or a positive tuberculosis skin test or interferon-gamma release assay (2 points), focal neurological deficit (1 point), cranial nerve palsy (1 point), and altered consciousness (1 point). A maximum of 4 points is allocated to CSF criteria including CSF clear appearance, cells count between 10 and 500 per $\mu$, lymphocytic predominance $(>50 \%)$, protein level greater than $1 \mathrm{~g} / \mathrm{L}$, and CSF to plasma glucose ratio of less than $50 \%$ or an absolute CSF glucose concentration less than $2 \mathrm{mmol} / \mathrm{L}$. Each item corresponds to 1 point. A maximum of 6 points is allocated to cerebral imaging criteria which includes the following items: hydrocephalus (1 point), basal meningeal enhancement (2 points), tuberculoma (2 points), infarct (1 point), and precontrast basal hyperdensity (1 point). A maximum of 4 points is allocated to evidence of tuberculosis elsewhere criteria which include the following items: chest radiograph suggestive of active tuberculosis (2 points of signs of tuberculosis and 4 points if miliary tuberculosis), evidence of tuberculosis outside the central nervous system on computed tomography, MRI, or ultrasound (2 points), evidence of MTB by detection of AFB or by culture from extraneural specimen (4 points), and positive nucleic acid amplification test for MTB from extraneural specimen (4 points). Patients with total score $\geq 10$ points (when cerebral imaging is not available) or $\geq 12$ points (when cerebral imaging is available) were considered as having a probable TBM. Patients with total score between 6 and 9 points (when cerebral imaging is not available) or score between 6 and 11 points (when cerebral imaging is available) were considered as having possible TBM.
2.3. Data Collection. Demographic, clinical, laboratory, and imaging data were collected from patient records. Clinical data include clinical signs at presentation. The duration of symptoms is defined as the time elapsed between the onset of signs and hospitalization. The time to diagnosis is the duration between admission to hospital and the start of antituberculosis therapy. Clinical severity at admission was assessed by using the British Medical Research Council (BMC) staging for TBM [8]. Grade 1 corresponds to alert and oriented patient without focal neurological deficits. Grade 2 is defined as a Glasgow coma scale of 11 to 14 or 15 with focal neurological deficits. Grade 3 is defined as a Glasgow coma scale of 10 or less with or without focal neurological deficits. Thwaites' diagnostic index (TDI) [9] was assessed for each case. This scoring system allows differentiating TB meningitis from bacterial meningitis. TDI is obtained by adding the score for age ( 0 if age $<36$ years and +2 if age $\geq 36$ years), for length of history ( 0 if $<6$ days and -5 if $\geq 6$ days), for peripheral blood white cells count (WCC) (0 if $<15,000$ per $\mathrm{mm}^{3}$ and +4 if $\geq 15,000$ per $\mathrm{mm}^{3}$ ), for CSF total WCC ( 0 if $<900$ per $\mathrm{mm}^{3}$ and +3 if $\geq 900$ per $\left.\mathrm{mm}^{3}\right)$, and for CSF neutrophils percentage ( 0 if $<75 \%$ and +4 if $\geq 75 \%$ ). A total score $\leq 4$ predicts the diagnosis of TBM and a total score $>4$ predicts the diagnosis of bacterial meningitis with a sensitivity of $86 \%$ and a specificity of $79 \%$. Laboratory data include CSF and other microbiological findings (sputum and gastric aspirate smear for AFB). Radiological findings include chest X-ray and cerebral imaging when available.

2.4. Statistical Analysis. Categorical variables were compared using Chi-square test or Fischer's exact test where appropriate. Predictors of mortality were assessed using logistic regression analysis. Univariate analysis was done to identify variables associated with mortality. Thereby, variables identified in univariate analysis with $p$ value $<0.2$ were entered into a multivariate logistic regression model to identify independent mortality risk factors. A $p$ value $<0.05$ was considered as significant. Statistical analyses were performed using Stata 12.0 (StataCorp LP, Texas, USA).

\section{Results}

3.1. Clinical Findings. A total of 75 patients were included in this study. Among them, 8 patients (10.7\%) had definite TBM, 44 (58.7\%) had probable TBM, and 23 (30.6\%) had possible TBM. The mean age was $35.4 \pm 12.7$ years (range 16-71 years). Forty-two patients (56\%) were male and 33 (44\%) were female. All patients were screened for HIV and only 3 patients (4\%) were HIV positive. Five patients $(6.7 \%)$ had tuberculosis history including 3 pulmonary tuberculosis and 2 extrapulmonary tuberculosis. Three patients (4\%) reported close contact with person with active tuberculosis. Twelve patients (16\%) had received BCG vaccination. Median duration of symptoms prior to admission was 3 weeks (IQR: $2-5)$. Duration of symptoms was $<1$ week for 2 patients (2.7\%), between 1 and 4 weeks for 37 patients (49.3\%), and $\geq 4$ weeks for 36 patients (48\%). Median time to diagnosis was 5 days (IQR: $3-8$ ). Time to diagnosis was $<3$ days for 13 patients (17.3\%), between 3 and 7 days for 38 patients (50.7\%), 
TABLE 1: Clinical findings on admission in patients with TBM.

\begin{tabular}{lc}
\hline Clinical findings & $n(\%)$ \\
\hline Fever & $72(96.0)$ \\
Headache & $58(77.3)$ \\
Weight loss & $33(44.0)$ \\
Persistent cough ( $\geq 2$ weeks) & $22(29.3)$ \\
Photophobia & $7(9.3)$ \\
Seizures & $19(25.3)$ \\
Neck stiffness & $52(69.3)$ \\
Altered consciousness & $55(73.3)$ \\
Coma & $6(8.0)$ \\
Cranial nerve palsy & $13(17.3)$ \\
Focal neurological signs & $8(10.7)$ \\
BMC & \\
$\quad$ Grade 1 & $13(17.3)$ \\
Grade 2 & $54(72.0)$ \\
Grade 3 & $8(10.7)$ \\
\hline
\end{tabular}

and $\geq 7$ days for 24 patients (32\%). TDI could be assessed for 68 patients. All patients had a score consistent with the diagnosis of TBM according to TDI (score $\leq 4)$. Among them, $29(42.6 \%)$ had TDI of -5 (maximal score for the diagnosis of TBM according to TDI). Clinical findings on admission are detailed in Table 1.

3.2. CSF Findings. CSF had clear appearance in all cases. Median CSF WCC was 75 per $\mathrm{mm}^{3}$ (IQR: 29-175). However, CSF WCC was $<10$ per $\mathrm{mm}^{3}$ in 8 cases $(10.7 \%)$. CSF lymphocytes percentage could be assessed in 72 cases. It was $<25 \%$ in 8 cases (11.1\%), between $25 \%$ and $50 \%$ in 15 cases $(20.8 \%)$, and between $50 \%$ and $75 \%$ in 11 cases (15.3\%). CSF lymphocyte predominance $(\geq 75 \%)$ was found in 38 cases $(52.8 \%)$. CSF glucose level was available for 74 patients. Median CSF glucose level was $1.48 \mathrm{mmol} / \mathrm{L}$ (IQR: $0.76-2.55)$. CSF glucose level was $<1.1 \mathrm{mmol} / \mathrm{L}$ in 26 cases (35.1\%), between 1.1 and $2.2 \mathrm{mmol} / \mathrm{L}$ in 26 cases $(35.1 \%$ ), and $\geq 2.2 \mathrm{mmol} / \mathrm{L}$ in 22 cases $(29.8 \%)$. CSF protein level was available for 74 patients. Median CSF protein level was $1 \mathrm{~g} / \mathrm{L}$ (IQR: 0.5-2.4). CSF protein level was normal $(<0.5 \mathrm{~g} / \mathrm{L})$ in 16 cases $(21.6 \%)$, between 0.5 and $1 \mathrm{~g} / \mathrm{L}$ in 11 cases $(14.9 \%)$, and $\geq$ $1 \mathrm{~g} / \mathrm{L}$ in 47 cases $(63.5 \%)$.

3.3. Radiological Findings. Sixty-eight patients (90.7\%) had chest X-ray. Chest radiological exam was abnormal in 43 cases $(63.2 \%)$. Major abnormalities were parenchymal infiltration $(n=25,36.8 \%)$, miliary pattern $(n=18,26.5 \%)$, pleural effusion $(n=4,5.9 \%)$, and cavitary lesions $(n=1,1.5 \%)$. Fifteen patients (20\%) had brain computed tomography scan. Seven patients $(46.7 \%)$ had an abnormal finding. Major findings were hydrocephalus $(n=5,71.4 \%)$ and basal enhancement $(n=3,60 \%)$.

3.4. Microbiological Findings. Among the 75 patients, only 13 patients $(17.3 \%)$ had a microbiological examination of the CSF for MTB identification. MTB were identified in the CSF samples of 8 patients. CSF culture was positive in 3 patients.
NAA test for MTB from CSF sample was positive for one patient. CSF culture and NAA test for MTB were both positive in 4 patients. AFB were identified from sputum in 9 patients (12\%).

3.5. Outcome. Antituberculosis therapy was started after diagnosis of TBM according to national guidelines. All patients except 5 patients who had tuberculosis history received the first-line regimen including rifampicin, isoniazid, ethambutol, and pyrazinamide during the intensive phase of the treatment ( 2 months). These four drugs were administered by oral route. Before 2012, patients received ethambutol and isoniazid for 6 months during the continuation phase of the treatment. After 2012, patients received rifampicin and isoniazid for 4 months during the continuation phase of the treatment. The 5 patients with tuberculosis history received rifampicin, isoniazid, ethambutol, pyrazinamide, and streptomycin during 2 months followed by continuation phase including rifampicin, isoniazid, ethambutol, and pyrazinamide.

Twenty-one (28\%) out of the 75 patients died. Median time to death was 8 days (IQR: 6-15) after admission to the hospital. Table 2 details univariate analysis of variables associated with inpatient mortality. Factors associated with inpatient mortality were age $\geq 35$ years $(p=0.044)$ and coma $(p=0.048)$. In multivariate analysis by logistic regression model $\left(p=0.0109, R^{2}=0.22\right)$, age $\geq 35$ years (adjusted odds ratio [aOR] 4.06, 95\% confidence interval [95\% CI] $1.16-14.26, p=0.029$ ) and coma (aOR 12.98, 95\% CI 1.13$149.16, p=0.040$ ) predicted inpatient mortality (Table 3).

\section{Discussion}

TBM remains an extremely serious disease. Most of the patients in our study $(82.7 \%)$ presented with advanced clinical stage (BMC grades 2 and 3). Several studies had reported similar observations [10, 11]. Moreover, we found that median duration of symptoms prior to admission was 3 weeks and only 2 patients out of 75 had less than a week of duration of symptoms. Duration of symptom prior to admission reported in previous studies was commonly longer than one week which is consistent with a subacute meningitis [12-14]. However, duration of symptoms prior to admission in our study was substantially longer than reported in these studies. Apart from these clinical features, the lack of health coverage and the weakness of the healthcare system lead patients to delay their admission due to the high cost of hospitalization which must be fully assumed by the patients themselves. This can partially explain the high proportion of patients who presented with advanced stage in our study.

TBM often occurred in patients without predisposing conditions in our study. Only 3 patients had HIV infection and 5 patients had history of tuberculosis. However, in African countries, rate of HIV infected patients among those with TBM is extremely high. Marais et al. [15] reported $88 \%$ of HIV infected patients among patients with TBM in African setting. This difference may be related to low prevalence of HIV in Madagascar. Indeed, the World Health Organization estimated HIV prevalence to 264 per 100,000 population 
TABLE 2: Univariate analysis of variables associated with inpatient mortality in TBM.

\begin{tabular}{|c|c|c|c|}
\hline Variable & $\begin{array}{c}\text { Survived } \\
n(\%) \\
54(72.0)\end{array}$ & $\begin{array}{c}\text { Died } \\
n(\%) \\
21(28.0)\end{array}$ & $p$ value \\
\hline Age $\geq 35$ years & $22(40.7)$ & $14(66.7)$ & 0.044 \\
\hline Male gender & $28(51.8)$ & $14(66.7)$ & 0.246 \\
\hline HIV infected & $2(3.7)$ & $1(4.8)$ & 0.633 \\
\hline Symptoms prior to admission $\geq 4$ weeks & $24(44.4)$ & $12(57.1)$ & 0.323 \\
\hline Time to diagnosis $\geq 7$ days & $18(33.3)$ & $6(28.6)$ & 0.691 \\
\hline BCG vaccination & $11(20.4)$ & $1(4.8)$ & 0.090 \\
\hline TB history & $3(5.6)$ & $2(9.5)$ & 0.432 \\
\hline Weight loss & $21(38.9)$ & $12(57.1)$ & 0.153 \\
\hline Cough $\geq 2$ weeks & $16(29.6)$ & $6(28.6)$ & 0.928 \\
\hline Photophobia & $5(9.3)$ & $2(9.5)$ & 0.972 \\
\hline Seizures & $16(29.6)$ & $3(14.3)$ & 0.140 \\
\hline Neck stiffness & $37(68.5)$ & $15(71.4)$ & 0.806 \\
\hline Altered consciousness & $41(75.9)$ & $14(66.7)$ & 0.416 \\
\hline Coma & $2(3.7)$ & $4(19.1)$ & 0.048 \\
\hline Cranial nerve palsy & $11(20.4)$ & $2(9.5)$ & 0.224 \\
\hline Focal neurological signs & $6(11.1)$ & $2(9.5)$ & 0.604 \\
\hline CSF WCC $\geq 150$ per $\mathrm{mm}^{3}$ & $16(29.6)$ & $11(52.4)$ & 0.065 \\
\hline CSF glucose level $\geq 1.1 \mathrm{mmol} / \mathrm{L}$ & $37(69.8)$ & $11(52.4)$ & 0.157 \\
\hline CSF protein level $\geq 1 \mathrm{~g} / \mathrm{L}$ & $31(58.5)$ & $16(76.2)$ & 0.154 \\
\hline Active pulmonary TB & $5(9.3)$ & $4(19.1)$ & 0.241 \\
\hline
\end{tabular}

TABLE 3: Multivariate analysis of factors associated with inpatient mortality.

\begin{tabular}{lccc}
\hline Variable & aOR & $95 \%$ CI & $p$ value \\
\hline Age $\geq 35$ years & 4.06 & $1.16-14.26$ & 0.029 \\
BCG vaccination & 0.19 & $0.02-1.99$ & 0.165 \\
Weight loss & 2.43 & $0.65-9.11$ & 0.187 \\
Seizures & 0.35 & $0.06-1.98$ & 0.233 \\
Coma & 12.98 & $1.13-149.16$ & 0.040 \\
CSF WCC $\geq 150$ per mm ${ }^{3}$ & 2.31 & $0.61-8.68$ & 0.217 \\
CSF protein level $\geq 1 \mathrm{~g} / \mathrm{L}$ & 2.84 & $0.72-11.14$ & 0.135 \\
CSF glucose level $\geq 1.1 \mathrm{mmol} / \mathrm{L}$ & 1.72 & $0.38-7.69$ & 0.478 \\
\hline
\end{tabular}

in 2014 [16]. Moreover, in high income countries with low prevalence of tuberculosis, TBM as well as tuberculosis in general often occur in patients coming from high prevalence countries $[13,17,18]$. Apart from HIV infection, other predisposing conditions including tuberculosis history, intravenous drug use, diabetes mellitus, alcoholism, or various host immunosuppression factors were commonly found in studies conducted in high income countries $[19,20]$. The profile of patients with TBM in our study is probably influenced by high burden of tuberculosis contrasting with low prevalence of HIV. Thereby, TBM tends to occur in patients without predisposing factors. However, we noted that only $16 \%$ of the patients had received BCG vaccination. Although the protective role of BCG vaccination against TBM in children is well known [21], its effect in adults remains uncertain [22].
However, Aronson et al. [23] and recently Nguipdop-Djomo et al. [24] demonstrated long-term efficacy of BCG vaccination against tuberculosis in general. Nevertheless, the proportion of vaccinated patients among those with TBM is currently low in setting applying the same strategy for BCG vaccine as in Madagascar [5, 12].

In resource-constrained setting, as in Madagascar, diagnosis of TBM remains a challenge which considerably contributes to diagnostic delay and underdiagnosis. In fact, CSF microbiological examination for MTB including detection of $\mathrm{AFB}$, culture, or NAA test were only done for 13 patients (17.3\%) due to intermittent access to these diagnostic tests. In all cases, the diagnosis of TBM was based on clinical features and CSF findings. Indeed, these features could help to differentiate TBM from other etiology of meningitis. Previous study demonstrated that clinical features including duration of symptoms $>5$ days, presence of headache, and CSF findings including CSF WCC $<1000$ per $\mathrm{mm}^{3}$, CSF clear appearance, CSF lymphocytes $>30 \%$, and CSF protein level help to distinguish TBM from acute bacterial meningitis with sensitivity of $93 \%$ and specificity of $77 \%$ when three or more of these features are present [25]. TDI allowed differentiating bacterial meningitis from TBM based on 5 criteria weighted by a score including age, duration of symptoms, peripheral blood WCC, CSF WCC, and CSF percentage of neutrophils [9]. Assessment of TDI in different studies including nonHIV adults patients showed sensitivity from $95.6 \%$ to $99 \%$ and a specificity from $70.8 \%$ to $80 \%[26,27]$. However, specificity is too low when test was applied in high HIV prevalence setting [28]. We retrospectively applied this diagnostic index 
in patients included in this study. All patients had a score consistent with the diagnosis of TBM. TDI could be useful in diagnosing TBM in our setting with low HIV prevalence and probably need further assessment.

In our study, patients received antituberculosis therapy following national guidelines and received 8-month or 6month regimen. All forms of tuberculosis including TBM are treated with the same regimen and duration. However, most of international guidelines including current WHO guideline recommend longer course of treatment ranging from 9 to 12 months [29-31]. Furthermore, short-term therapy is not supported by strong evidence [32]. However, neither the length nor the optimal treatment regimen is well known in TBM.

Overall mortality in our study was $28 \%$. Mortality rate in our setting is considerably lower than reported in other African countries where mortality ranged from $59.9 \%$ to 87.9\% [4]. This difference is likely due to lower HIV prevalence in our setting as HIV infection is a major prognosis factor among TBM patients [33]. However, our study has important limitation due to its retrospective nature which may contribute to underestimating long-term mortality and sequelae. In fact, patient follow-up is not always possible. After discharge from hospital, patients are referred to primary care setting near to their home to promote adherence to antituberculosis therapy. In our study, age $\geq 35$ was identified as an independent predictor of mortality. Previous studies also found that older patients had higher risk of death compared to younger patients with different threshold compared to our finding [34-36]. This difference is likely due to age distribution. Nevertheless, Shaw et al. [37] demonstrated a linear relationship between age and risk of death. Apart from age, coma was also found to be an independent risk of death in our study. Several studies reported similar observations $[34,38]$. In our study, death occurred within 8 days following admission. Although we failed to demonstrate relationship between treatment delay and mortality, previous study showed that patients receiving antituberculosis therapy within 1 day of initial presentation had better prognosis compared with patients receiving antituberculosis therapy after 1 day. Likewise, patients who started antituberculosis therapy immediately or after nonspecific antibiotics within 5 days had better prognosis [39]. In our setting, most of the patients received empiric antimicrobial therapy before antituberculosis therapy since lack of clinical improvement with nonspecific antimicrobial therapy is considered as an evidence for the diagnosis of TBM. Indeed, time to diagnosis was $\geq 3$ days in most of the cases. However, this approach tends to postpone initiation of appropriate antituberculosis therapy.

Several limitations were identified due to the retrospective design of this study. We were unable to assess long-term prognosis of patients. Some biological data such as blood glucose were not always available and did not allow assessing CSF to blood glucose ratio for the majority of the patients. CSF microbiological examination for MTB as well as cerebral imaging was limited and not routinely performed because of its cost which was entirely borne by the patient. However, this retrospective study allowed describing what was done in daily clinical practice.

\section{Conclusion}

In this study, most of patients with TBM presented with advanced stages at admission. HIV infection rate as well as BCG vaccination rate among TBM patients remained low. Clinical features were mostly consistent with subacute meningitis. CSF examination showed moderate pleocytosis with lymphocytic predominance, low glucose level, and increased protein level in the majority of cases. Microbiological confirmation of TBM was low due to poor access to culture or molecular test for MTB identification. Mortality rate was $28 \%$ and death generally occurred early after admission. Age $\geq 35$ years and coma were identified as independent predictors of mortality. In resource-constrained settings, the diagnosis of TBM should be based promptly on clinical findings and CSF features as microbiological confirmation test are not always available. Moreover, antituberculosis therapy should be started immediately whenever clinical and CSF findings are consistent with the diagnosis of TBM to improve outcome.

\section{Ethical Approval}

This study was retrospective and ethical approval was not required.

\section{Conflicts of Interest}

The authors declare that they have no conflicts of interest.

\section{References}

[1] WHO, Global Tuberculosis Report 2013, WHO, Geneva, Switzerland, 2013.

[2] M. Phypers, T. Harris, and C. Power, "CNS tuberculosis: a longitudinal analysis of epidemiological and clinical features," International Journal of Tuberculosis and Lung Disease, vol. 10, no. 1, pp. 99-103, 2006.

[3] CDC, Reported Tuberculosis in the United States, 2013, CDC, Atlanta, Ga, USA, 2014.

[4] Y. W. Woldeamanuel and B. Girma, "A 43-year systematic review and meta-analysis: case-fatality and risk of death among adults with tuberculous meningitis in Africa," Journal of Neurology, vol. 261, no. 5, pp. 851-865, 2014.

[5] J. Kalita, U. K. Misra, and P. Ranjan, "Predictors of long-term neurological sequelae of tuberculous meningitis: a multivariate analysis," European Journal of Neurology, vol. 14, no. 1, pp. 33-37, 2007.

[6] J. Ho, B. J. Marais, G. L. Gilbert, and A. P. Ralph, "Diagnosing tuberculous meningitis-have we made any progress?” Tropical Medicine and International Health, vol. 18, no. 6, pp. 783-793, 2013.

[7] S. Marais, G. Thwaites, J. F. Schoeman et al., "Tuberculous meningitis: a uniform case definition for use in clinical research," The Lancet Infectious Diseases, vol. 10, no. 11, pp. 803-812, 2010.

[8] R. B. Rock, M. Olin, C. A. Baker, T. W. Molitor, and P. K. Peterson, "Central nervous system tuberculosis: pathogenesis and clinical aspects," Clinical Microbiology Reviews, vol. 21, no. 2, pp. 243-261, 2008. 
[9] G. E. Thwaites, T. T. H. Chau, K. Stepniewska et al., "Diagnosis of adult tuberculous meningitis by use of clinical and laboratory features," Lancet, vol. 360, no. 9342, pp. 1287-1292, 2002.

[10] E. G. Miftode, O. S. Dorneanu, D. A. Leca et al., “Tuberculous meningitis in children and adults: a 10-year retrospective comparative analysis," PLOS ONE, vol. 10, no. 7, Article ID e0133477, 2015.

[11] P. N. Sutlas, A. Unal, H. Forta, S. Senol, and D. Kirbas, "Tuberculous meningitis in adults: review of 61 cases," Infection, vol. 31, no. 6, pp. 387-391, 2003.

[12] S. Hosoglu, M. F. Geyik, I. Balik, B. Aygen, S. Erol, and S. G. Aygencel, "Tuberculous meningitis in adults in Turkey: epidemiology, diagnosis, clinic and laboratory," European Journal of Epidemiology, vol. 18, no. 4, pp. 337-343, 2003.

[13] A.-S. H. Christensen, Å. B. Andersen, V. Ø. Thomsen, P. H. Andersen, and I. S. Johansen, "Tuberculous meningitis in Denmark: a review of 50 cases," BMC Infectious Diseases, vol. 11, article 47, 2011.

[14] N. E. Anderson, J. Somaratne, D. F. Mason, D. Holland, and M. G. Thomas, "A review of tuberculous meningitis at Auckland City Hospital, New Zealand," Journal of Clinical Neuroscience, vol. 17, no. 8, pp. 1018-1022, 2010.

[15] S. Marais, D. J. Pepper, C. Schutz, R. J. Wilkinson, and G. Meintjes, "Presentation and outcome of tuberculous meningitis in a high HIV prevalence setting," PLoS ONE, vol. 6, no. 5, Article ID e20077, 2011.

[16] WHO, World Health Statistics 2014, WHO, Geneva, Switzerland, 2014.

[17] L. Fenner, S. Gagneux, P. Helbling et al., "Mycobacterium tuberculosis transmission in a country with low tuberculosis incidence: role of immigration and HIV infection," Journal of Clinical Microbiology, vol. 50, no. 2, pp. 388-395, 2012.

[18] E. Svensson, J. Millet, A. Lindqvist, M. Olsson, M. Ridell, and N. Rastogi, "Impact of immigration on tuberculosis epidemiology in a low-incidence country," Clinical Microbiology and Infection, vol. 17, no. 6, pp. 881-887, 2011.

[19] B. Roca, N. Tornador, and E. Tornador, "Presentation and outcome of tuberculous meningitis in adults in the province of Castellon, Spain: a retrospective study," Epidemiology and Infection, vol. 136, no. 11, pp. 1455-1462, 2008.

[20] C. Bidstrup, P. H. Andersen, P. Skinhøj, and Å. B. Andersen, "Tuberculous meningitis in a country with a low incidence of tuberculosis: still a serious disease and a diagnostic challenge," Scandinavian Journal of Infectious Diseases, vol. 34, no. 11, pp. 811-814, 2002.

[21] B. B. Trunz, P. Fine, and C. Dye, "Effect of BCG vaccination on childhood tuberculous meningitis and miliary tuberculosis worldwide: a meta-analysis and assessment of cost-effectiveness," Lancet, vol. 367, no. 9517, pp. 1173-1180, 2006.

[22] P. Mangtani, I. Abubakar, C. Ariti et al., "Protection by BCG vaccine against tuberculosis: a systematic review of randomized controlled trials," Clinical Infectious Diseases, vol. 58, no. 4, pp. 470-480, 2014.

[23] N. E. Aronson, M. Santosham, G. W. Comstock et al., "Longterm efficacy of BCG vaccine in american indians and alaska natives: a 60-year follow-up study," JAMA, vol. 291, no. 17, pp. 2086-2091, 2004.

[24] P. Nguipdop-Djomo, E. Heldal, L. C. Rodrigues, I. Abubakar, and P. Mangtani, "Duration of BCG protection against tuberculosis and change in effectiveness with time since vaccination in Norway: a retrospective population-based cohort study," The Lancet Infectious Diseases, vol. 16, no. 2, pp. 219-226, 2016.
[25] F. G. Youssef, S. A. Afifi, A. M. Azab et al., "Differentiation of tuberculous meningitis from acute bacterial meningitis using simple clinical and laboratory parameters," Diagnostic Microbiology and Infectious Disease, vol. 55, no. 4, pp. 275-278, 2006.

[26] M. Sunbul, A. Atilla, S. Esen, C. Eroglu, and H. Leblebicioglu, "Thwaites' diagnostic scoring and the prediction of tuberculous meningitis," Medical Principles and Practice, vol. 14, no. 3, pp. 151-154, 2005.

[27] M. E. Török, H. D. T. Nghia, T. T. H. Chau et al., "Validation of a diagnostic algorithm for adult tuberculous meningitis," American Journal of Tropical Medicine and Hygiene, vol. 77, no. 3, pp. 555-559, 2007.

[28] A. M. Checkley, Y. Njalale, M. Scarborough, and E. E. Zjilstra, "Sensitivity and specificity of an index for the diagnosis of TB meningitis in patients in an urban teaching hospital in Malawi," Tropical Medicine and International Health, vol. 13, no. 8, pp. 1042-1046, 2008.

[29] WHO, Treatment of Tuberculosis: Guidelines, WHO, Geneva, Switzerland, 4th edition, 2010.

[30] G. Thwaites, M. Fisher, C. Hemingway, G. Scott, T. Solomon, and J. Innes, "British Infection Society guidelines for the diagnosis and treatment of tuberculosis of the central nervous system in adults and children," Journal of Infection, vol. 59, no. 3, pp. 167-187, 2009.

[31] American Thoracic Society, CDC, and Infectious Diseases Society of America, "Treatment of tuberculosis," MMWR Recommendations and Reports, vol. 52, no. RR-11, pp. 1-77, 2003.

[32] S. Jullien, H. Ryan, M. Modi, and R. Bhatia, "Six months therapy for tuberculous meningitis," The Cochrane Database of Systematic Reviews, no. 9, Article ID CD012091, 2016.

[33] G. E. Thwaites, N. D. Bang, N. H. Dung et al., "The influence of HIV infection on clinical presentation, response to treatment and outcome in adults with tuberculous meningitis," Journal of Infectious Diseases, vol. 192, no. 12, pp. 2134-2141, 2005.

[34] K. K. Yasar, F. Pehlivanoglu, and G. Sengoz, "Predictors of mortality in tuberculous meningitis: a multivariate analysis of 160 cases," International Journal of Tuberculosis and Lung Disease, vol. 14, no. 10, pp. 1330-1335, 2010.

[35] E. L. George, T. Iype, A. Cherian et al., "Predictors of mortality in patients with meningeal tuberculosis," Neurology India, vol. 60, no. 1, pp. 18-22, 2012.

[36] P. C. Hsu, C. C. Yang, J. J. Ye, P. Y. Huang, P. C. Chiang, and M. $\mathrm{H}$. Lee, "Prognostic factors of tuberculous meningitis in adults: a 6-year retrospective study at a tertiary hospital in northern Taiwan," Journal of Microbiology, Immunology and Infection, vol. 43, no. 2, pp. 111-118, 2010.

[37] J. E. T. Shaw, J. G. Pasipanodya, and T. Gumbo, "Meningeal tuberculosis: high long-term mortality despite standard therapy," Medicine, vol. 89, no. 3, pp. 189-195, 2010.

[38] S. Hosoglu, M. F. Geyik, I. Balik et al., "Predictors of outcome in patients with tuberculous meningitis," International Journal of Tuberculosis and Lung Disease, vol. 6, no. 1, pp. 64-70, 2002.

[39] J.-J. Sheu, R.-Y. Yuan, and C.-C. Yang, "Predictors for outcome and treatment delay in patients with tuberculous meningitis," The American Journal of the Medical Sciences, vol. 338, no. 2, pp. 134-139, 2009. 


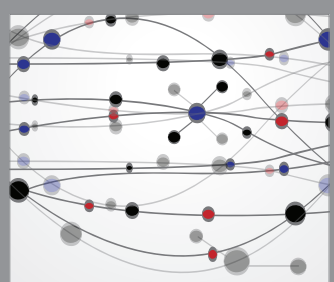

The Scientific World Journal
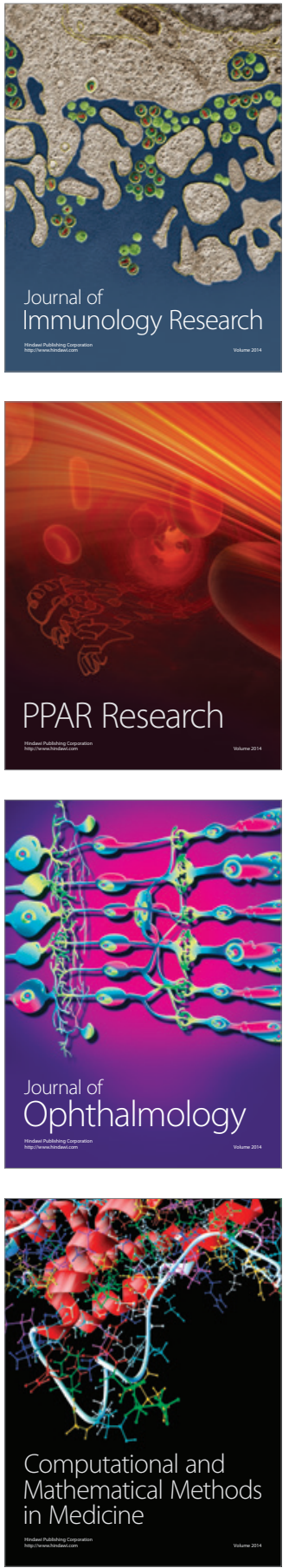

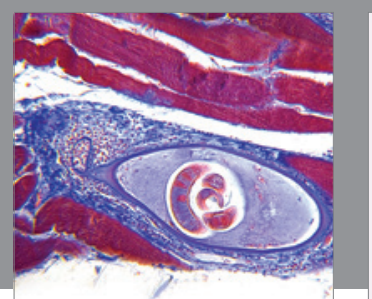

Gastroenterology Research and Practice
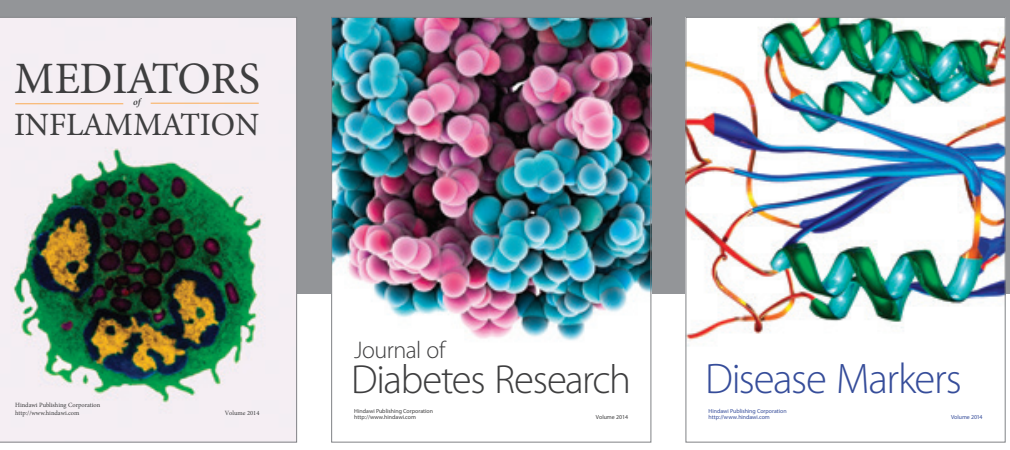

Disease Markers



\section{Hindawi}

Submit your manuscripts at

https://www.hindawi.com
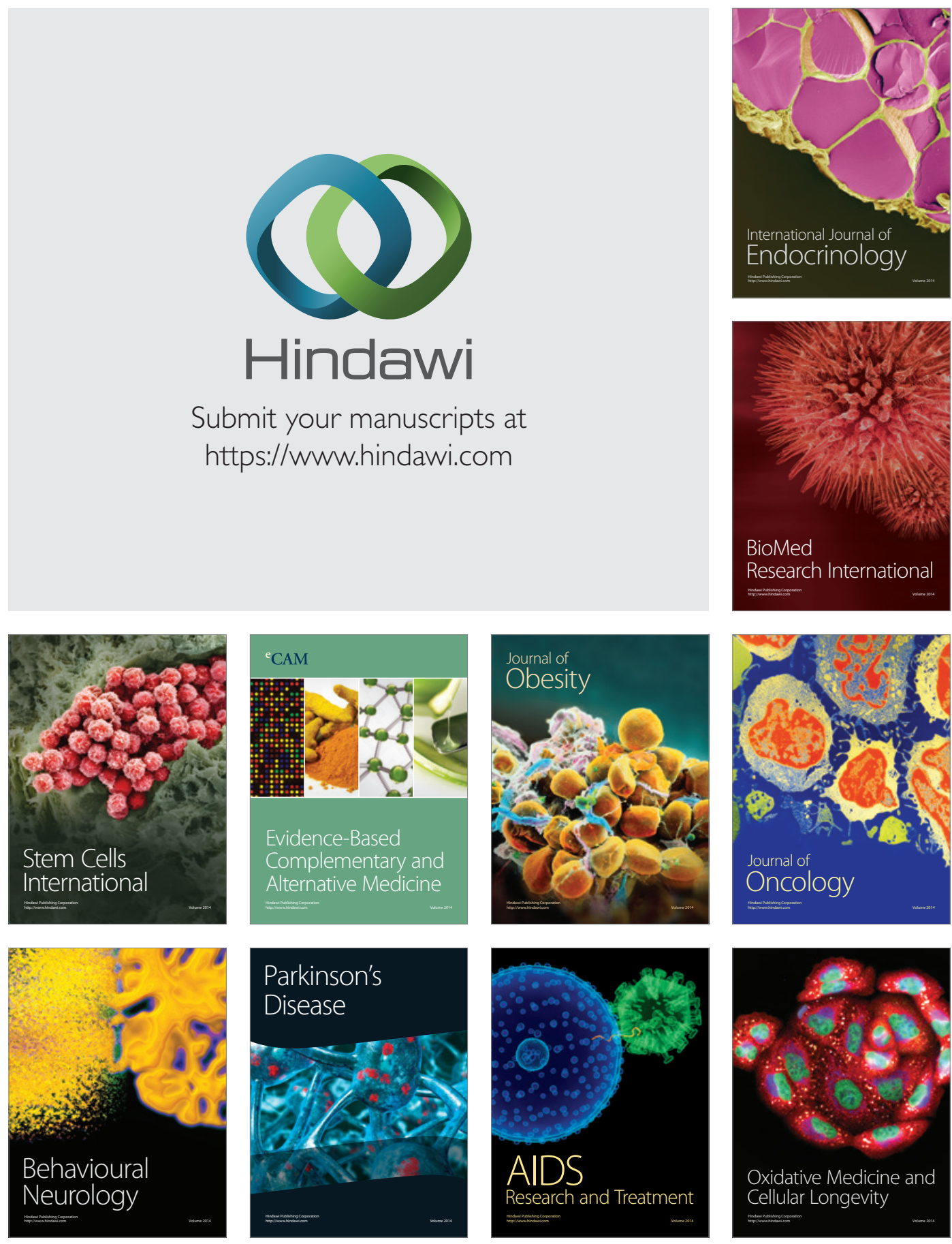